\title{
Stability and dynamics of Pt-Si liquid microdroplets on $\mathrm{Si}(001)$
}

\author{
W.-C. Yang, H. Ade, and R. J. Nemanich* \\ Department of Physics, North Carolina State University, Raleigh, North Carolina 27695-8202, USA \\ (Received 7 May 2003; revised manuscript received 10 September 2003; published 29 January 2004)
}

\begin{abstract}
The formation and dynamics of Pt-Si liquid droplets on $\mathrm{Si}$ (001) surfaces are explored with real-time ultraviolet photoelectron emission microscopy. PtSi islands of micrometer lateral diameter begin to melt and are transformed into molten Pt-Si alloy islands below the melting point of bulk PtSi. In particular, at $\sim 1100{ }^{\circ} \mathrm{C}$ surface migration of the liquid microdroplets is observed, where the droplets move directionally from the cold to the hot regions of the surface following the temperature gradient across the substrate. It is proposed that the droplet surface migration is due to dissolution-diffusion-deposition flow of Si through the droplet driven by the $\mathrm{Si}$ concentration difference in the droplet. In addition, the migration rate of the droplet is measured as a function of temperature and droplet diameter. Above a minimum diameter, the migration velocity is independent of the droplet size, which indicates that Si diffusivity through the droplet is the primary factor determining the rate of migration. The activation energy for the Si diffusion in the droplet is found to be $\sim 0.57 \mathrm{eV}$. We conclude that the thermal and chemical stability of the droplet-substrate interface significantly affects the evolution and dynamics of the liquid island on the surface.
\end{abstract}

DOI: 10.1103/PhysRevB.69.045421

PACS number(s): 68.08.- p, 68.37.Nq

\section{INTRODUCTION}

It is well known that thin metal films on $\mathrm{Si}$ react to form an equilibrium silicide-Si interface and higher-temperature annealing transforms the thin film into silicide island structures. ${ }^{1}$ For some metal-Si $(M-\mathrm{Si})$ systems there is a eutectic at a composition between that of the silicide and $\mathrm{Si}$ (i.e., $\mathrm{Au}, \mathrm{Al}, \mathrm{Pd}, \mathrm{Ni}$, and $\mathrm{Pt}$ ). For these systems the metallic silicide islands will be in a liquid state at temperatures above the eutectic temperature.

Moreover, $M$-Si liquid islands on $\mathrm{Si}$ surfaces have been employed for the fabrication of self-assembled nanostructures using the vapor-liquid-solid (VLS) mechanism. ${ }^{2-4}$ For instance, it has been reported that $\mathrm{Si}$ nanowires (or whiskers) can be grown by silane exposure of $\mathrm{Au}-\mathrm{Si}$ droplets on $\mathrm{Si}$ (Ref. 3). Here, the nanoscale Au-Si droplets act as the transport medium of $\mathrm{Si}$, and the growth occurs at the interface of the liquid drop and the substrate.

The surface and interface stability of liquid $M$-Si droplets on Si will affect the evolution and dynamics of the droplets. Unlike other solid heterointerfaces, the interface between a $M$-Si molten island and the Si substrate is affected by chemical equilibrium conditions due to temperature dependence of the Si concentration of the $M-\mathrm{Si}$. Thus, to control the development of the surface morphology, it will be necessary to develop an understanding of the droplet interface stability, droplet formation and evolution mechanisms, and effects that can lead to dynamical interactions.

In this study we explore these aspects for the Pt-Si system. PtSi thin films exhibit both excellent Ohmic as well as Schottky barrier characteristics with $p$ - and $n$-type silicon, respectively, and thus are used for contact metallization in silicon integrated circuit technology. ${ }^{5}$ The reaction of Pt film on Si leads to a stable PtSi compound for annealing at above $600{ }^{\circ} \mathrm{C}$ (Ref. 6). Further annealing at above $800{ }^{\circ} \mathrm{C}$ induces island structures. The eutectic between $\mathrm{PtSi}$ and $\mathrm{Si}$ is at a composition of $\sim 67 \% \mathrm{Si}$ in which the $\mathrm{Pt}-\mathrm{Si}$ liquid phase will form at a temperature $\sim 980{ }^{\circ} \mathrm{C}$ (Ref. 7).
Liquid droplets on a solid surface can be induced to migrate via Brownian motion and/or under external forces, which can be created by gradients in surface tension or concentration. $^{8-13}$ Droplet migration is important in a variety of coalescence processes. The migration of a $M$-Si liquid droplet on a $\mathrm{Si}$ surface is a complex phenomenon because the equilibrium composition of the droplets varies with temperature. From a fundamental point of view, $M$-Si droplet migration also involves a mass transport mechanism and chemical equilibrium at the liquid-solid interface. ${ }^{14-17}$ Previously, the electromigration and thermomigration of various metallic droplets on Si surfaces have been described in terms of metal diffusion into the Si substrate. ${ }^{13}$ However, without in situ, real-time measurements of these complicated processes, the theoretical aspects cannot be developed to consistently explain the observations.

In this study, UV photoelectron emission microscopy (UV-PEEM) is employed to investigate the formation and dynamics of Pt-Si microdroplets. The PEEM technique allows real-time observation and direct imaging during surface processes with exceptional surface sensitivity and high resolution. ${ }^{18}$ Previously, real-time PEEM measurements have explored the coarsening of nanoscale $\mathrm{TiSi}_{2}$ islands on a $\mathrm{Si}$ surface at high temperature ${ }^{19}$ and the migration of $\mathrm{Au}$ liquid droplets on a Si surface. ${ }^{20}$ Therefore, PEEM is particularly suited for the measurement of dynamic processes on semiconductor surfaces. In PEEM, the image contrast originates from variation in the photoelectric yield, where the photoelectric threshold affects the image contrast. In metals, the photoelectric threshold is the work function while for semiconductors the threshold is dependent on the electron affinity, the band gap, the band bending, and the doping. ${ }^{21,22}$ In this study, the photothreshold difference of PtSi $(4.85 \mathrm{eV})$ and $\mathrm{Si}(>5.1 \mathrm{eV})$ is used to obtain image contrast.

Here, we report the stability and dynamics of Pt-Si microdroplets on a $\mathrm{Si}$ (001) surface. It is shown that the molten Pt-Si islands nucleate below the melting point of bulk PtSi. The molten islands are observed to migrate and grow 
through coalescence. Using PEEM, we monitor the microdroplet migration and droplet-droplet interactions while annealing at temperatures up to $\sim 1200^{\circ} \mathrm{C}$. The migration rates of the droplets are measured in detail as a function of droplet size and temperature. A model of the driving force for droplet migration is proposed. Our results suggest that Si diffusivity is the primary factor determining the migration rate of the droplets.

\section{EXPERIMENTAL DETAILS}

The experiments were performed in an UV-PEEM (Elmitec PEEM-III) system combined with the ultraviolet Free Electron Laser (UV-FEL) located at the Duke University Free Electron Laser Laboratory. This system allows realtime, high-resolution $(\sim 10 \mathrm{~nm})$ imaging with the sample at elevated temperature $\left(>1200^{\circ} \mathrm{C}\right)$. The base pressure of the PEEM system was below $2 \times 10^{-10}$ Torr. The electric potential used for accelerating the imaging electrons is approximately $20 \mathrm{kV}$ across a gap of $2 \mathrm{~mm}$.

The incident UV light is obtained from either a $100-\mathrm{W} \mathrm{Hg}$ discharge lamp with an upper cutoff energy near $5.0 \mathrm{eV}$ or the tunable spontaneous emission of the UV-FEL with a photon energy of $4.9 \mathrm{eV}$. The spontaneous radiation of the FEL is pulsed with a repetition rate of $15 \mathrm{MHz}$ and a pulse duration of $\sim 300 \mathrm{psec}^{23}$ The average power of the UV light was $\sim 200 \mu \mathrm{W}$, and the corresponding peak power was $\sim 2 \mathrm{~W}$. The focused beam was elliptical in shape with a size of $\sim 20 \mu \mathrm{m} \times 100 \mu \mathrm{m}$. This corresponds to an average power density of $\sim 0.1 \mathrm{~W} / \mathrm{cm}^{2}$. The capabilities of the PEEM-FEL are described in more detail elsewhere. ${ }^{18}$

Silicon (001) wafers ( $n$ type, P doped, resistivity $0.8-1.2$ $\Omega \mathrm{cm}, 25 \mathrm{~mm}$ diameter) were employed as the substrates. The wafers were cleaned first by UV-ozone exposure and then by an HF-based spin etch to remove hydrocarbon contaminants and the native oxide layers. ${ }^{24}$ After ex situ cleaning, the wafers were loaded into a UHV-MBE chamber (base pressure of $1 \times 10^{-10}$ Torr). Prior to Pt deposition, the wafers were annealed at a temperature of $900^{\circ} \mathrm{C}$ for $10 \mathrm{~min}$ by filament radiation from the backside. The residual oxide and hydrogen were removed by this heat treatment. Following the annealing, low-energy electron diffraction (LEED) displayed a $2 \times 1$ diffraction pattern characteristic of the $\mathrm{Si}(001)$ reconstructed surface, and Auger electron spectroscopy (AES) data indicated no detectable carbon or oxygen.

Platinum was deposited from an $e$-gun source onto the clean Si substrate at room temperature, and the thickness was monitored with a quartz crystal monitor. A 10-nm Pt film was deposited at a rate of $0.05 \mathrm{~nm} / \mathrm{sec}$. In addition, to observe island migration on $\mathrm{Si}$ surfaces without Pt coverage, an array of $10-\mathrm{nm}$-thick Pt dots of $100-\mu \mathrm{m}$ in diameter with $500-\mu \mathrm{m}$ spacing was deposited through a shadow mask onto a $\mathrm{Si}$ (001) surface.

Sections of the sample $\left(9 \times 9 \mathrm{~mm}^{2}\right)$ were mounted to a sample holder and then introduced into the PEEM chamber. To observe the reaction process of the $\mathrm{Pt}$ thin films, the samples were heated by filament radiation $\left(<800^{\circ} \mathrm{C}\right)$ and electron bombardment $\left(>800^{\circ} \mathrm{C}\right)$ from the backside of the sample holder while the surfaces were imaged by PEEM.
The temperature of the samples was controlled by a thermocouple attached to the sample holder. The actual temperature of the surface was measured with a finely focused (spot size of $1 \mathrm{~mm}$ diameter) optical pyrometer.

PEEM images are enhanced with a microchannel plate and displayed on a phosphor screen. The images were observed with a charge-coupled-device (CCD) camera and stored digitally with an image processor and also captured on videotape. For the data presented, 16 successive images were integrated, where the resulting images correspond to an integrated signal of $16 / 30$ of a second. However, for the droplet velocity measurements, single-frame images (corresponding to $1 / 30$ of a second) were obtained from the videotape recording. The recorded images were digitized at a rate of one per 15 secs. The rate was limited by digitization rate of the image processor.

After the substrates were unloaded from the PEEM, ex situ atomic force microscopy (AFM) and scanning tunneling microscopy (SEM) were performed to compare the surface morphology with the PEEM images. In addition, the composition of the droplets and substrate surface were investigated by micro-Raman spectroscopy with a focused beam diameter of $\sim 2 \mu \mathrm{m}$.

\section{RESULTS AND DISCUSSION}

\section{A. Formation of Pt-Si liquid microdroplets}

Figure 1 represents a series of PEEM images of a 10-nmthick Pt film deposited on a $\mathrm{Si}(001)$ during annealing up to $960^{\circ} \mathrm{C}$. At $800^{\circ} \mathrm{C}$, the initial uniform emission surface [Fig. 1(a) ] changed into a surface with unstructured bright features on a darker background [Fig. 1(b)]. It is known that the solid-state reaction of $\mathrm{Pt}$ films on $\mathrm{Si}$ leads to the formation of $\mathrm{PtSi}$ at $\sim 600^{\circ} \mathrm{C}$ (Ref. 6) and that a uniform PtSi layer trans-

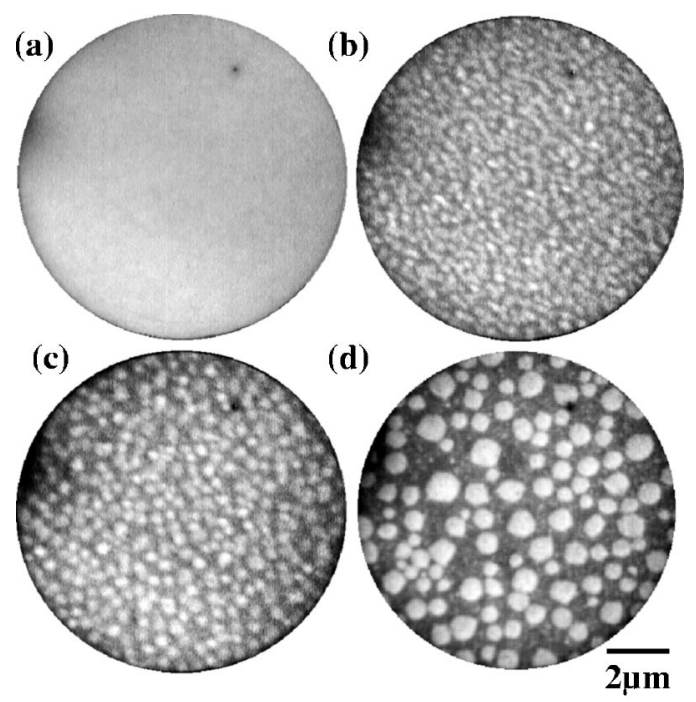

FIG. 1. PEEM images of a Pt film of $10 \mathrm{~nm}$ deposited on $\mathrm{Si}(001)$ substrate. The images were obtained at annealing temperatures of (a) $600{ }^{\circ} \mathrm{C}$, (b) $800{ }^{\circ} \mathrm{C}$, and (c), (d) $960{ }^{\circ} \mathrm{C}$, respectively. The sequential images of (c), (d) were taken 1 min apart. The incident light is the spontaneous emission radiation from the UV-FEL with a photon energy of $4.9 \mathrm{eV}$, and the field of view is $10 \mu \mathrm{m}$. 
forms into island structures at $\sim 800^{\circ} \mathrm{C}$. Thus, the bright features correspond to $\mathrm{PtSi}$ islands while the darker regions are identified as the exposed Si surface. We can exclude the effect of surface oxidation of the 10-nm Pt film on Pt silicide formation since the oxidation of Pt films suppresses significantly the silicide formation only for films of a few nanometer thicknesses. ${ }^{25}$

As the temperature was increased, the islands grew larger through ripening and coalescence with neighboring islands, resulting in well-separated island structures as shown in Fig. 1(c). However, a significant transition in the shape and lateral size of the islands was observed at $\sim 960^{\circ} \mathrm{C}$. The islands with an average diameter of $\sim 400 \mathrm{~nm}$ [Fig. 1(c)] transformed spontaneously into larger islands of $\sim 1 \mu \mathrm{m}$ in diameter [Fig. 1(d)]. During this process, some of the $\sim 400-\mathrm{nm}$ islands disappeared at their positions via apparent island collisions or ripening. Also, the larger $\sim 1-\mu \mathrm{m}$ islands exhibited a smoother circumference and surface. The shape of the islands remained constant at higher temperature up to $\sim 1150^{\circ} \mathrm{C}$. The transition process apparently corresponds to a phase transition of the solid islands into liquid droplets. The $\sim \mu \mathrm{m}$-diameter islands appear to be in the liquid state as may be expected since this temperature is close to the PtSi-Si eutectic temperature $\left(\sim 980^{\circ} \mathrm{C}\right)$ (Ref. 7). This abrupt transition, which occurs in about $1 \mathrm{~min}$ in our measurements, indicates that the PtSi islands initiate melting at a temperature near the eutectic temperature. (Our temperature uncertainty is $\pm 40{ }^{\circ} \mathrm{C}$.)

In a separate experiment the island formation was detected by LEED measurements. In these measurements, the sample was annealed to a specific temperature, cooled to near room temperature and transferred to the LEED chamber. For the initial Pt deposition and for annealing up to $600^{\circ} \mathrm{C}$, no LEED was observed. After annealing at $\sim 750^{\circ} \mathrm{C}$, the LEED showed a mixed $c(4 \times 2)$ and $c(4 \times 6)$ diffraction pattern, which is characteristic of the reconstructed $\mathrm{Si}$ (001) surface induced by a coverage of $1 / 4$ monolayer of Pt (Ref. 26). The appearance of this LEED pattern indicates the exposure of substrate regions presumably due to the formation of PtSi islands. It also indicates that Pt atoms can diffuse on the Si surface between the islands.

The nucleation of Pt-Si liquid droplets below the melting point of $\mathrm{PtSi}\left(\sim 1230^{\circ} \mathrm{C}\right)$ can be explained with reference to the Pt-Si binary phase diagram (Fig. 2) (Ref. 7). The eutectic between PtSi and $\mathrm{Si}$ is at a composition of $\sim 67 \% \mathrm{Si}$ and a temperature of $\sim 980^{\circ} \mathrm{C}$. Thus the formation of molten Pt-Si at the eutectic temperature requires excess $\mathrm{Si}$ to be incorporated into the PtSi islands. We propose that the islands initiate melting from the island-substrate interface where excess $\mathrm{Si}$ from the substrate and the PtSi would lead to a Pt-Si liquid droplet with the eutectic composition. Additional Si from the substrate would continue to diffuse into the island until it is completely molten. The Pt-Si droplet is then in thermodynamic equilibrium with the $\mathrm{Si}$ substrate.

According to this model, at higher temperatures, the composition of the Pt-Si liquid droplets would follow the liquidus between the eutectic and Si up to the Si melting temperature of $\sim 1414^{\circ} \mathrm{C}$. This would be accomplished by diffusion

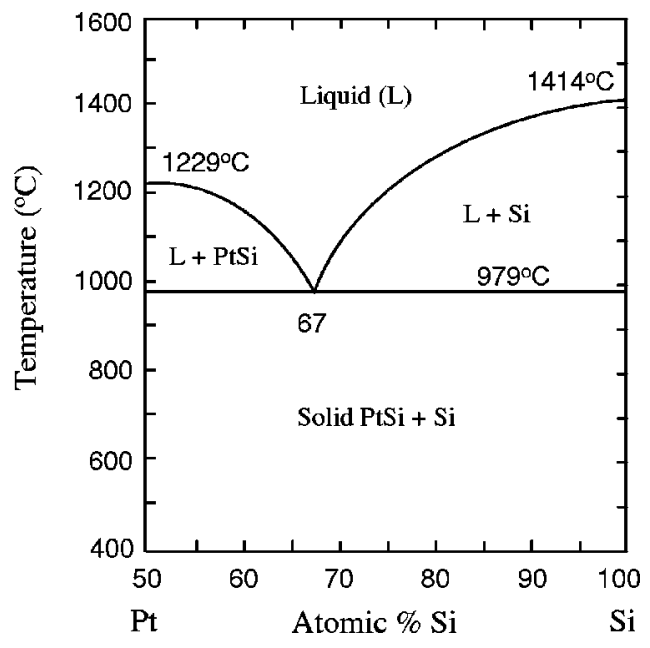

FIG. 2. A portion of Pt-Si binary phase diagram (Ref. 7).

of Si into the droplet as the temperature is increased. This diffusion across the interface is necessary to maintain equilibrium with the $\mathrm{Si}$ substrate.

To explore this effect, we employed micro-Raman spectroscopy to analyze a relatively large droplet after rapid solidification. A sample was prepared as described above and annealed to $1150{ }^{\circ} \mathrm{C}$ for several minutes. The sample was then rapidly cooled by shutting off the substrate heating with a cooling rate of $\sim 900^{\circ} \mathrm{C} / 3 \mathrm{~min}$ and removed from vacuum for micro-Raman analysis. The Raman spectrum of an $\sim 10-\mu \mathrm{m}$-diam droplet is displayed in Fig. 3. The scan exhibits characteristic features at $\sim 140$ and $520 \mathrm{~cm}^{-1}$ which are attributed to crystalline PtSi and $\mathrm{Si}$, respectively. ${ }^{27}$ The micro-Raman spot size is $\sim 2 \mu \mathrm{m}$, and the light penetration depth in the metallic silicide is expected to be less than 100 $\mathrm{nm}$. For the $2-10-\mu \mathrm{m}$-diam islands, the height of the islands above the surface is in the range of $190-540 \mathrm{~nm}$, as determined by AFM. Therefore, the scan represents the properties of the island and not the substrate. The Si peak is attributed

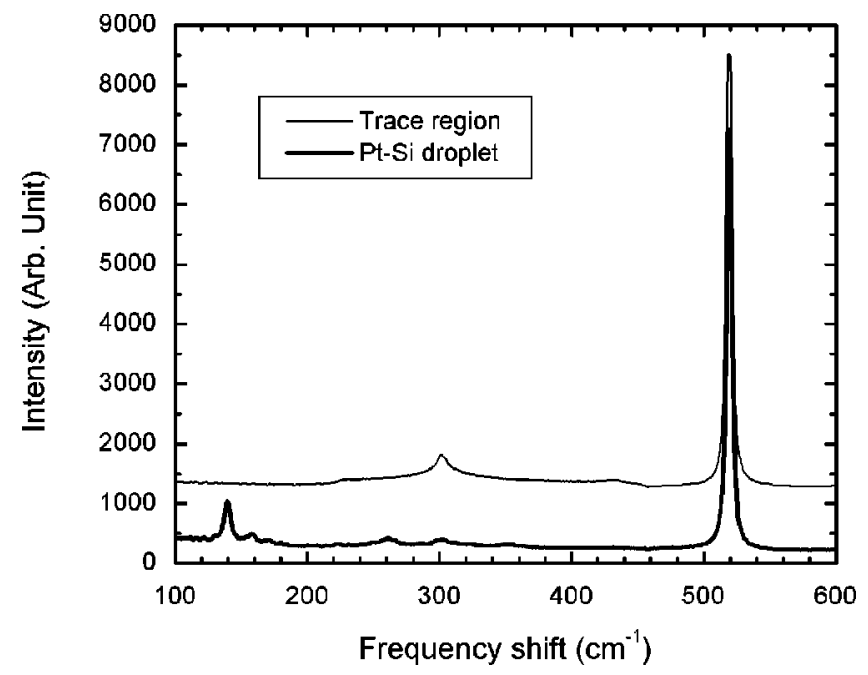

FIG. 3. Micro-Raman spectra from a $10-\mu \mathrm{m}$ droplet and the trace region left behind the droplet. Spectra were obtained after rapid cooling of the droplet. 


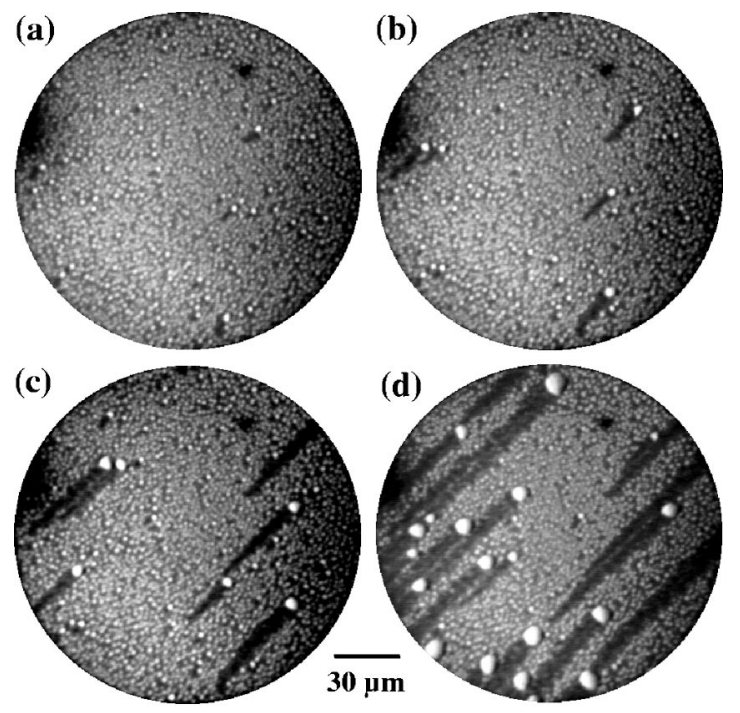

FIG. 4. A time sequence of PEEM images of droplet migration on a Si surface during annealing at $1100{ }^{\circ} \mathrm{C}$ for (a) $0 \mathrm{sec}$, (b) $15 \mathrm{sec}$, (c) $45 \mathrm{sec}$, and (d) $75 \mathrm{sec}$, respectively. The field of view for the images is $150 \mu \mathrm{m}$, and images were excited with a Hg-discharge lamp.

to excess $\mathrm{Si}$ that has segregated from the PtSi during the solidification process. We note that the Raman signal from $\mathrm{PtSi}$ is quite weak compared to that of $\mathrm{Si}$. The excess $\mathrm{Si}$ originates from the $\mathrm{Si}$ dissolved from the substrate to achieve chemical equilibrium, suggesting that the Pt-Si liquid droplet would be a Si-rich Pt-Si alloy.

\section{B. Dynamics of droplet migration}

It is evident that the islands are in a liquid state above the eutectic temperature. Figures 4 and 5 present PEEM images of the island dynamics during annealing at $1100^{\circ} \mathrm{C}$. Shown in Fig. 4 is a time sequence of PEEM images of the initiation of island migration. When droplets grow larger than $\sim 2 \mu \mathrm{m}$ in diameter via coalescence and ripening, some droplets initiate surface migration. As time progresses, the moving islands grow larger through coalescence with stationary smaller islands. The coalescence process is shown in Figs. 5(a) and 5(b), where it is observed that a relatively larger island migrates across the surface while most of the smaller islands remain in place. As the annealing time progresses, the larger island collects stationary islands of $\sim 1 \mu \mathrm{m}$ and continues to grow during its traversing of the surface. At a larger field of view [Figs. 5(c) and 5(d)], some of the moving islands are observed to coalesce with neighboring islands and to reshape to form larger circular islands.

The dark stripe left behind a moving island is the trace, which has the emission character of the Si substrate. MicroRaman measurements were obtained from the trace region, and the Raman spectra of the droplet trace (Fig. 3) showed features at 300 and $520 \mathrm{~cm}^{-1}$ without the feature at $140 \mathrm{~cm}^{-1}$. The features at 520 and $300 \mathrm{~cm}^{-1}$ are first and second Raman scattering of crystalline $\mathrm{Si}$, respectively. ${ }^{27}$

Upon close examination it was found that the droplets migrate essentially parallel to each other and in one direction

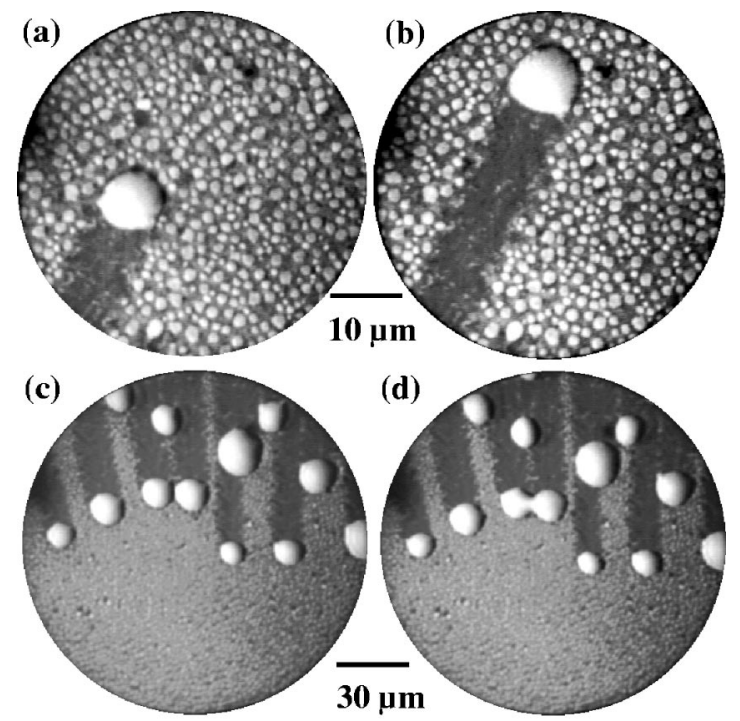

FIG. 5. A series of PEEM images of liquid island migration on Si surfaces during annealing at $1100{ }^{\circ} \mathrm{C}$ for (a) $0 \mathrm{sec}$ and (b) $20 \mathrm{sec}$, respectively. The time interval between (c) and (d) is $15 \mathrm{sec}$. The field of view for the images is $50 \mu \mathrm{m}$ and $150 \mu \mathrm{m}$ in (a),(b) and (c),(d), respectively. All images were obtained with the sample at $1100{ }^{\circ} \mathrm{C}$. The incident light is from a $\mathrm{Hg}$-discharge lamp.

[Figs. 5(c) and 5(d)]. Through ex situ optical microscopy of a rapidly cooled sample, it was found that the droplets migrated from the edge towards the center of the substrate. Our in situ observations were then able to verify this observation.

We considered various effects that could contribute to the observed migration. To exclude effects due to the optical interactions and the electric field of the microscope, we repeated the experiments in the PEEM but without the applied electric field $(100 \mathrm{kV} / \mathrm{cm})$ and without UV-light illumination. The PEEM images obtained after processing exhibited evidence of island migration similar to the real-time PEEM measurements, indicating that the high electric field or the UV light does not affect the island dynamics.

We next explored the effect of temperature. Using an optical pyrometer, the temperature of different areas of the substrate was measured during the annealing of the sample. The temperature at the center of the substrate was $\sim 50{ }^{\circ} \mathrm{C}$ higher than at the edge (the distance between the center and edge is $\sim 3 \mathrm{~mm}$ ). This radial temperature difference on the sample surface indicates that the droplets moved toward the hotter region of the sample, and we have concluded that the temperature gradient of the substrate is responsible for the droplet migration.

To explore the motion of droplets on a Si surface free of $\mathrm{Pt}$ and without the effects of coalescence, an array of 10-nmthick Pt dots of $100 \mu \mathrm{m}$ in diameter with $500 \mu \mathrm{m}$ spacing was deposited on a $\mathrm{Si}$ (001) surface at room temperature. During annealing of the film at $\sim 1080{ }^{\circ} \mathrm{C}$, various diameter droplets formed in the $\mathrm{Pt}$ dot region via droplet coalescence, and migration of larger droplets occurs as with the continuous films [Fig. 6(a)]. Moreover, these droplets continued to migrate beyond the edge of the original dot and traveled over the Si surface regions that were free of Pt. Migration over the 
(a)

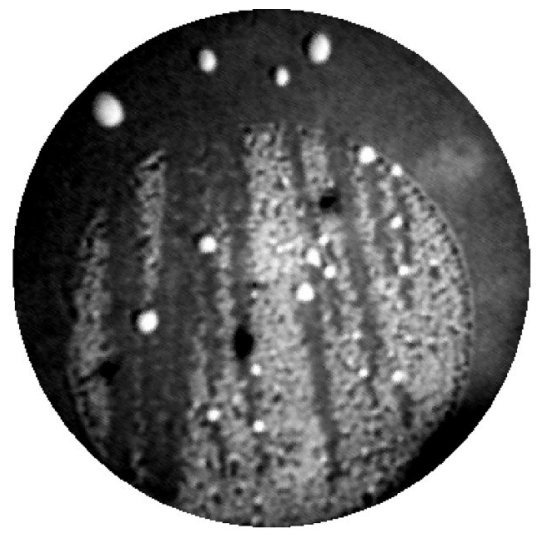

(b)

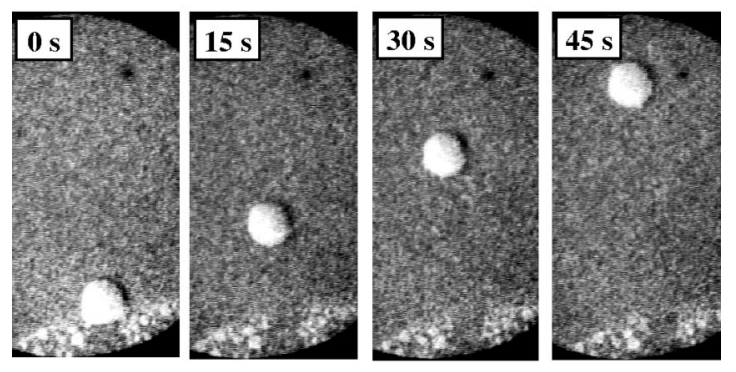

FIG. 6. (a) PEEM image of a 100- $\mu \mathrm{m}$ Pt circle on $\mathrm{Si}(001)$. The droplets originate from within the Pt dot and travel through the Pt-free region. (b) A time sequence of PEEM images showing droplet migration over the $\mathrm{Pt}$-free $\mathrm{Si}$ surface. The images were obtained at a temperature of $1085^{\circ} \mathrm{C}$ when the droplet was leaving the Pt dot edge at $t=0$. Each single frame image was taken $15 \mathrm{sec}$ apart. The size of the droplet is $6.3 \mu \mathrm{m}$, and the mean velocity is $0.7 \mu \mathrm{m} / \mathrm{sec}$. The field of view are $150 \mu \mathrm{m}$ and $50 \mu \mathrm{m}$, in (a) and (b), respectively.

bare $\mathrm{Si}$ is not affected by coalescence with other islands and more clearly reflects the dynamics of the migration. For a more detailed measurement of the droplet velocity, the migration distance and diameter of each droplet were analyzed using single-frame images from the real-time videotape recording. An example of the velocity measurement is shown in Fig. 6(b). Each image was obtained $15 \mathrm{sec}$ apart. As a droplet traversed the Si surface, the velocity of the droplet in each time separation remained essentially constant. The diameter and shape of the moving droplets also appears to remain constant.

Using the above method, the average migration velocity was measured at various annealing temperatures as a function of the droplet diameter. The quantitative results are summarized in Fig. 7. The velocity is nearly independent of droplet diameter while at each temperature there is a slight tendency for the velocity to increase with droplet diameter in the measured range of $1.5-7.0 \mu \mathrm{m}$. This relationship was observed very distinctly for the droplets in a large number of samples.

The results also seem to indicate a minimum diameter for the initial island migration. To observe the temperature of initial motion of a droplet, a sample was prepared with migrating micro droplets (at $\sim 1100^{\circ} \mathrm{C}$ ). The sample was then cooled to $1000^{\circ} \mathrm{C}$, where all island migration ceased. As the temperature was increased, the temperature of initial migra-

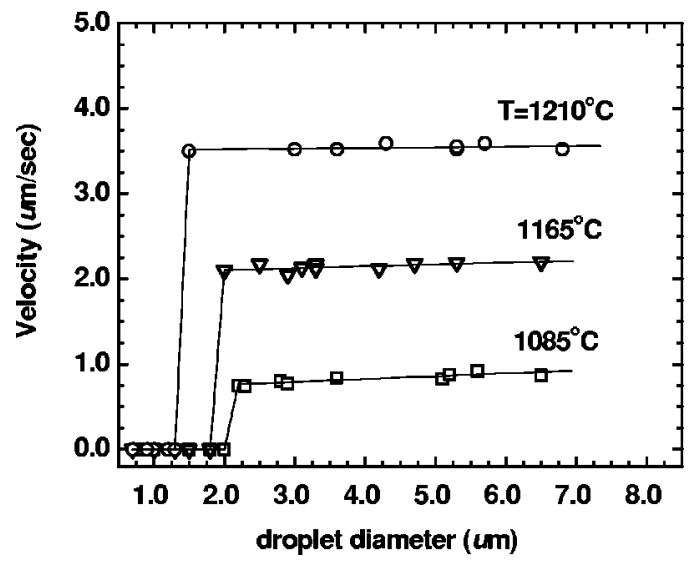

FIG. 7. The migration velocity of Pt-Si droplets vs diameter for droplets migrating on a Pt-free Si (001) surface at various anneal temperatures.

tion of islands of different diameter was recorded. It was found that smaller islands required a higher temperature to initiate migration. For example, the migration of a $\sim 2-\mu \mathrm{m}$ droplet was detected at $1085^{\circ} \mathrm{C}$ while a $\sim 1.5-\mu \mathrm{m}$ droplet initiated migration at $\sim 1200^{\circ} \mathrm{C}$ (Fig. 7).

\section{Model for surface migration of the droplets}

It is evident that the thermal gradient, $\boldsymbol{\nabla} T$ around the droplet is responsible for the migration of the Pt-Si microdroplets. We consider various temperature-dependent effects that could contribute to the migration.

First, the $\boldsymbol{\nabla} T$ could generate a variation in the surface tension $\gamma$ of the liquid droplets. We note that the tangential component of the surface tension gradient of a liquid droplet gives rise to Marangoni flow, which drives the droplet toward the region of high surface tension. ${ }^{9}$ For a Pt-Si alloy liquid, $\gamma$ may be dependent on the concentration of $\mathrm{Si}$ in the droplet which will depend on the temperature. We can determine the sign of the temperature gradient of the surface tension, $d \gamma / d T=(d \gamma / d C)(d C / d T)$ where $C$ is the Si concentration in the Pt-Si liquid. Since $d \gamma / d C<0$ (Ref. 28) and $d C / d T>0$ (Ref. 7), then $d \gamma / d T$ is negative. In other words, the increase of $\mathrm{Si}$ in the Pt-Si liquid with increasing temperature leads to a decrease in surface tension. Thus, the surface tension of the droplet would be lowest on the side of the droplet that is the hottest. As a result, the Pt-Si droplet would be expected to migrate toward the colder region-i.e., toward higher surface tension. This is contrary to our observation.

Second, a variation of the substrate surface and interface energies could be induced by $\boldsymbol{\nabla} T$. This capillary force can also drive the droplets toward the region of lower surface and interface energies. ${ }^{10}$ To estimate the surface and interfacial energies of the droplet, the interface of the droplet and substrate was investigated by cross sectional SEM [Fig. 8(a)]. The solidified island extends below the $\mathrm{Si}$ surface. The penetration depth of the island into the surface is $370 \mathrm{~nm}$ and the height of island above the surface is $290 \mathrm{~nm}$. The degree of recession of the island into the substrate and the shape of the island are determined by the surface and interface energies, 
(a)

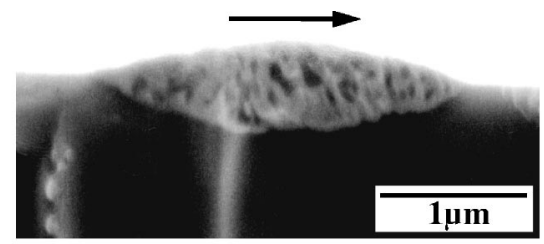

(b)

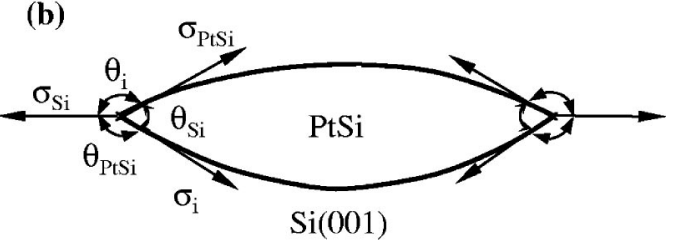

FIG. 8. (a) A cross-sectional SEM image of a rapidly cooled 2.5 - $\mu \mathrm{m}$-diam droplet. The cleavage was roughly through the center of the selected droplet. The arrow indicates the migration direction of the island. (b) A schematic drawing of the cross section of the island. Here $\sigma$ represents surface or interface energy per unit area and $\theta$ is the contact angle. The subscripts, $\mathrm{Si}, i$, and PtSi refer to the $\mathrm{Si}$ surface, the interface, and the PtSi surface, respectively.

which can be determined by the contact angle of the island with the substrate [Fig. 8(b)] (Ref. 29). The contact angles at both the advancing and receding interfaces were determined from the SEM photographs. Then, using an analysis based on a liquid-liquid model, ${ }^{29}$ the surface and interface energies were deduced from the surface tension balance equation. The results are summarized in Table I. It is noted that the velocity of the droplet driven by capillary force can be determined by the relationship

$$
V \propto \frac{d S}{d T} \frac{d T}{d z}
$$

where $S=\sigma_{\mathrm{Si}}-\left(\sigma_{i}+\sigma_{\mathrm{PtSi}}\right)$ and the $z$ direction is from a cold to a hot region. ${ }^{10}$ The $S$ value at the edge of the cold interface is larger than at the hot interface and then $d S / d T$ is positive. Thus, the droplet would be expected to migrate toward the cold region-i.e., toward a larger $S$ value. This is also contrary to our observation.

Third, we consider the diffusion of Si into and out of the droplet. To be in chemical equilibrium at the liquid-solid interface, the Pt-Si droplet can dissolve $\mathrm{Si}$ atoms from or deposit $\mathrm{Si}$ to the substrate across the droplet-substrate interface. We note again that at higher temperatures the $M-\mathrm{Si}$ droplet will have an increased Si concentration. ${ }^{7}$ Because of the temperature gradient, the $\mathrm{Si}$ equilibrium concentration in the droplet at the high-temperature interface front, $C_{h}$, will be greater than that at the low-temperature interface front,

TABLE I. The measured contact angles (in degrees) and the corresponding surface and interface energies in $\mathrm{erg} / \mathrm{cm}^{2}$. The $\mathrm{Si}$ surface energy was taken from Ref. 27.

\begin{tabular}{ccccccc}
\hline \hline Interface & $\theta_{\mathrm{Si}}$ & $\theta_{\mathrm{i}}$ & $\theta_{\mathrm{PtSi}}$ & $\sigma_{\mathrm{Si}}$ & $\sigma_{\mathrm{i}}$ & $\sigma_{\mathrm{PtSi}}$ \\
\hline Advancing & 41 & 165 & 154 & 1210 & 473 & 802 \\
Receding & 35 & 167 & 158 & 1210 & 471 & 784 \\
\hline \hline
\end{tabular}
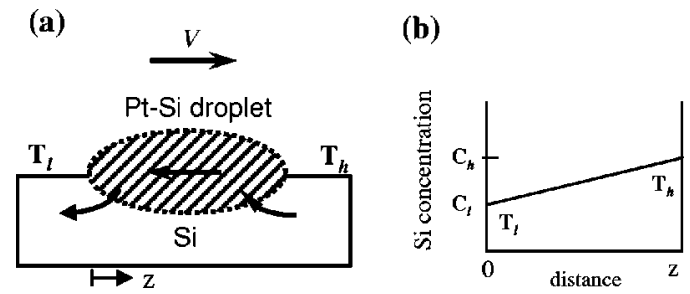

FIG. 9. (a) A schematic model for the migration of a Pt-Si droplet along a $\mathrm{Si}$ surface with a temperature gradient $\left(T_{h}>T_{l}\right)$. The arrows indicate the $\mathrm{Si}$ diffusion into and through the droplet. (b) The Si concentration profile in the droplet vs distance from back to front.

$C_{l}$. Consequently, a concentration gradient of $\mathrm{Si}, \nabla C$, is present in the liquid droplet. Through diffusion, the Si concentration in a droplet tends to a uniform value. As a result, supersaturation at the cold interface leads to deposition of $\mathrm{Si}$ onto the substrate while undersaturation of $\mathrm{Si}$ at the hot interface induces continuous dissolution of $\mathrm{Si}$ from the substrate. This process, which is shown schematically in Fig. 9, would cause the droplet's apparent center of mass to move continuously toward the hotter region of the $\mathrm{Si}$ substrate. Apparently, ex situ AFM and SEM measurements of the sample shown in Fig. 6 indicated that the trace region behind the droplet is slightly higher than the surrounding regions (not shown). The nonplanar top surface in the Si trace region would be expected to be a recrystallized Si layer. However, crystal defects and epitaxial deposition of $\mathrm{Si}$ in the trace region were not investigated in this experiment.

Based on this $\nabla C$ of $\mathrm{Si}$ in the Pt-Si droplet, the rate of droplet migration will be dependent on the rate of Si diffusion through the droplet or the rates of Si dissolution from and deposition onto the substrate. To maintain the circular shape and size during migration, the deposition rate at the cold interface of the droplet must be nearly balanced by the dissolution rate at the hot interface of the droplet. This appears to be the case for Pt-Si droplet migration. Also, the constant shape of the moving droplet indicates that the rate of dissolution and deposition is higher than the rate of the $\mathrm{Si}$ diffusion through the droplet. Thus, we propose that the migration rate of the droplet is limited primarily by the Si diffusivity through the liquid.

Prior analysis of molten droplets in bulk systems has established that the variation in the droplet velocity with the droplets size is related to the material transport mechanism in the droplet. ${ }^{11,15}$ If the migration rate is limited by the interface reactions (such as dissolution and deposition), then the migration velocity will vary with droplet size $(d), V$ $\propto f(T) d^{n}$. In contrast, if the volume diffusion through the droplet is the limiting mechanism of the migration, then the migration rate should be independent of droplet size, $V$ $\propto f(T)$ (Ref. 15). This appears to be the case for the Pt-Si alloy droplets where the $\mathrm{Si}$ diffusion through the droplet should be the primary rate-determining step.

As the annealing temperature is increased, the average velocity of the droplet increases rapidly (Fig. 7). Assuming that the Si diffusion in the liquid droplet is the rate-limiting step, an equation for the droplet velocity can be derived from 
TABLE II. The variation of the concentration gradient $[(d X / d T) \nabla T]$ and diffusivity $(D)$ of the droplet versus temperature. The variables $1 /(1-X)$ and $d X / d T$ were obtained from the Pt-Si binary phase diagram. The diffusivity $D$ was calculated with velocity equation.

\begin{tabular}{|c|c|c|c|c|c|c|}
\hline$T\left({ }^{\circ} \mathrm{C}\right)$ & $\begin{array}{c}V \\
(\mu \mathrm{m} / \mathrm{sec})\end{array}$ & $\begin{array}{c}\boldsymbol{\nabla} T \\
\left({ }^{\circ} \mathrm{C} / \mathrm{cm}\right)\end{array}$ & $1 /(1-X)$ & $\begin{array}{c}d X / d T\left({ }^{\circ} \mathrm{C}^{-1}\right) \\
\left(\times 10^{-4}\right)\end{array}$ & $\begin{array}{l}(d X / d T) \nabla T \\
\quad\left(\times 10^{-2}\right)\end{array}$ & $\begin{array}{c}D\left(\mathrm{~cm}^{2} / \mathrm{s}\right) \\
\left(\times 10^{-4}\right)\end{array}$ \\
\hline 1085 & $0.82 \pm 0.05$ & 138 & 3.37 & 4.16 & 5.74 & $4.24 \pm 0.26$ \\
\hline 1105 & $1.10 \pm 0.04$ & 143 & 3.50 & 5.06 & 7.24 & $4.35 \pm 0.16$ \\
\hline 1130 & $1.49 \pm 0.05$ & 148 & 3.62 & 5.44 & 8.05 & $5.11 \pm 0.17$ \\
\hline 1165 & $2.15 \pm 0.05$ & 155 & 3.94 & 6.36 & 9.86 & $5.54 \pm 0.13$ \\
\hline 1210 & $3.55 \pm 0.03$ & 165 & 4.52 & 7.56 & 12.47 & $6.29 \pm 0.05$ \\
\hline
\end{tabular}

the Si mass balance equation at the droplet-substrate interface. For Si-rich Al-Si droplets, Cline and Anthony have derived a velocity equation in terms of the $\mathrm{Si}$ atomic fraction in the droplet, ${ }^{17}$

$$
V=-\frac{D}{1-X} \frac{d X}{d T} \nabla T,
$$

where $D$ is the diffusivity of $\mathrm{Si}$ through the liquid droplet, $X$ is the $\mathrm{Si}$ atomic fraction in the liquid, $d X / d T$ is the atomic fraction change with respect to temperature (inverse liquidus slope), and $\boldsymbol{\nabla} T$ is the temperature gradient of the substrate. The variables $X, d X / d T$, and $D$ are temperature dependent.

To investigate the dominant factor affecting the increased velocity of $\mathrm{Pt}-\mathrm{Si}$ droplets with increasing temperature, we adopted the above velocity equation and estimated the variation for each factor. Although the velocity equation was deduced for the migration of a three-dimensional droplet in a three-dimensional (3D) solid, the expression is not dependent on shape. This expression should describe the surface migration of the Pt-Si liquid droplets, since the droplets penetrate into the substrate and have significant 3D character and the temperature gradient is predominantly along the surface. In our analysis the variables $X$ and $d X / d T$ were obtained from the liquidus curve of the Pt-Si binary phase diagram, and $D$ was calculated from the above velocity equation. The results are summarized in Table II. The dominant factor is $(d X / d T) \nabla T$, which is attributed to the concentration gradient in the liquid droplet. As the temperature increases, the dissolution of Si near the hot interface increases significantly, and the concentration gradient in the droplet would be likewise increased, which would enhance the $\mathrm{Si}$ transport rate. Thus, within this model the increase in droplet velocity with increasing temperature would be due to the enhancement of dissolution at the hot interface.

In general, diffusivity is dependent upon temperature, $D$ $=D_{0} \exp (-Q / k T)$, where $Q$ is the activation energy, $k$ is Boltzmann's constant, and $D_{0}$ is the preexponential factor, respectively. The activation energy can be obtained from the slope of the Arrhenius plot of $\ln (D)$ versus 1/T using the equation $Q=-k[\partial \ln D / \partial(1 / T)]$. For the Pt-Si liquid droplets, the plot of $\ln (D)$ versus $1 / T$ (Fig. 10) was obtained using the values of Table II. The slope of the plot obtained with curve fitting indicates an activation energy of $\sim 0.57$ $\pm 0.06 \mathrm{eV}$. This value is closer to that for diffusion of most liquid metals (0.1-0.4 eV) (Ref. 30) but less than that of solids (1-6 eV) (Ref. 31). In addition, the derived preexponential factor $D_{0}$ for the Pt-Si system is (5.49 \pm 0.17$)$ $\times 10^{-2} \mathrm{~cm}^{2} / \mathrm{sec}$, which is between that in liquid metal $\left(10^{-3}-10^{-4} \mathrm{~cm}^{2} / \mathrm{sec}\right.$ ) (Ref. 30) and in solids $\left(0.1-1 \mathrm{~cm}^{2} / \mathrm{sec}\right)$ (Ref. 31). This result implies that the activation energy required for diffusion of $\mathrm{Si}$ in our Pt-Si system is near that in a liquid-metal system and is lower than in solids.

One aspect of the experiment that cannot be readily explained with this model is the observed temperature and size dependence of the initial motion of the microdroplets. Within the model the droplet motion is determined by the interface reactions and the bulk diffusion. We would speculate that the size dependence of the initial migration may be related to the role of the interface reactions where these effects would become significantly for smaller dimensions and lower temperatures.

\section{SUMMARY}

In this study, we have used real-time PEEM to observe the nucleation and migration of Pt-Si liquid droplets. The microdroplets were formed below the melting temperature of $\mathrm{PtSi}$ and were determined to be a Si-rich Pt-Si alloy. Characteristic of liquid droplets, the islands grew through coalescence. The droplets moved from cold to hot regions on the surface following the temperature gradient of the substrate. We proposed that the migration of the droplets is a due to dissolution-diffusion-deposition flow of $\mathrm{Si}$ through the drop-

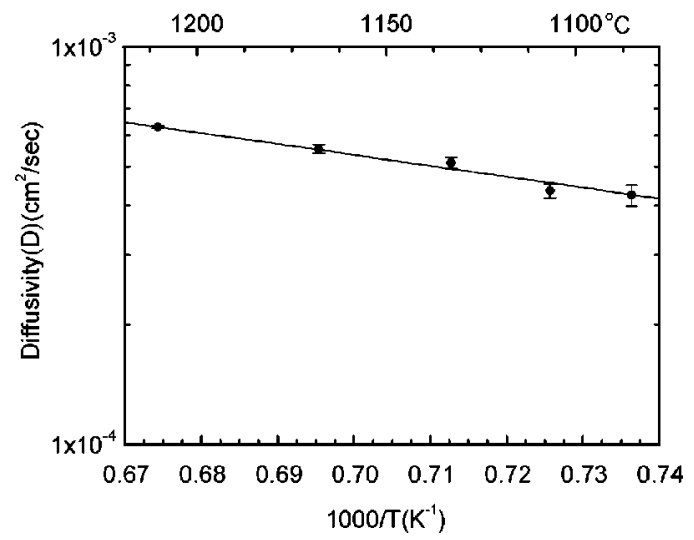

FIG. 10. An Arrhenius plot of $\ln (D)$ vs $1000 / T$ for the Pt-Si liquid droplets. The values were obtained from Table II. 
let produced by the thermal gradient. In addition, we found that the migration rate of the Pt-Si droplet is independent of the droplet diameter, and we deduced that Si diffusion is the primary factor which determines the migration rate of the Pt-Si droplets. The activation energy for diffusion of $\mathrm{Si}$ in the droplet was found to be $\sim 0.57 \mathrm{eV}$. It is concluded that droplet migration at elevated temperature may be a general phenomenon for all $M$-Si eutectic systems and that the thermal and chemical stability at the interface of $M$-Si droplets on $\mathrm{Si}$ surfaces plays a significant role in the surface evolution of droplets.

\section{ACKNOWLEDGMENTS}

We gratefully acknowledge the Duke Free Electron Laser Laboratory for access to the OK-4 free electron laser. This work was supported by the NSF under Grant No. DMR 0102652 and the DOD MFEL program as administered by the AFOSR through Contract No. F49620-00-1-0370.
*Electronic address: Robert_Nemanich@ncsu.edu

${ }^{1}$ E. Jiran and C.V. Thompson, Thin Solid Films 208, 23 (1992).

${ }^{2}$ Y. Wakayama and S. Tanaka, Surf. Sci. 420, 190 (1999).

${ }^{3}$ Y. Homma, P. Finnie, and T. Ogino, Appl. Phys. Lett. 74, 815 (1999).

${ }^{4}$ J. Westwater, D.P. Gosain, S. Tomiya, S. Usui, and H. Ruda, J. Vac. Sci. Technol. B 15, 554 (1997).

${ }^{5}$ M. P. Lepselter and J. M. Andrews, in Ohmic Contacts to Semiconductors, edited by B. Schwartz (Princeton University Press, Princeton, 1969).

${ }^{6}$ S.P. Murarka, E. Kinsbron, D.B. Fraser, J.M. Andrews, and E.J. Lloyd, J. Appl. Phys. 54, 6943 (1983); A.K. Sinha, R.B. Marcus, T.T. Sheng, and S.E. Haszko, ibid. 43, 3637 (1972).

${ }^{7}$ M. Hansen, Constitution of Binary Alloys (McGraw-Hill, New York, 1958).

${ }^{8}$ M.K. Chaudhury and G.M. Whitesides, Science 256, 1539 (1992).

${ }^{9}$ F. Brochard, Langmuir 5, 432 (1989).

${ }^{10}$ J.B. Brzoska, F. Brochard-Wyart, and F. Rondelez, Langmuir 9 , 2220 (1993).

${ }^{11}$ F.A. Nichols, J. Nucl. Mater. 30, 143 (1969).

${ }^{12}$ K. Ichimura, S. Oh, and M. Nakagawa, Science 288, 1624 (2000).

${ }^{13}$ T. Ichinokawa, H. Izumi, C. Haginoya, and H. Itoh, Phys. Rev. B 47, 9654 (1993).

${ }^{14}$ W.A. Tiller, J. Cryst. Growth 6, 77 (1969).

${ }^{15}$ P. Shewmon, Trans. Metall. Soc. AIME 230, 1134 (1964).
${ }^{16}$ W. G. Pfann, Zone Melting (Wiley, New York, 1966).

${ }^{17}$ H.E. Cline and T.R. Anthony, J. Appl. Phys. 47, 2325 (1976).

${ }^{18}$ H. Ade, W. Yang, S. English, J. Hartman, R.F. Davis, R.J. Nemanich, V.N. Litvinenko, I.V. Pinayev, Y. Wu, and J.M. Mady, Surf. Rev. Lett. 5, 1257 (1998).

${ }^{19}$ W.-C. Yang, M. Zeman, H. Ade, and R.J. Nemanich, Phys. Rev. Lett. 90, 136102 (2003).

${ }^{20}$ M. Mundschau, Surf. Sci. 213, 381 (1989).

${ }^{21}$ M. Giesen, R.J. Phaneuf, E.D. Williams, T.L. Einstein, and H. Ibach, Appl. Phys. A: Mater. Sci. Process. A64, 423 (1997).

${ }^{22}$ V.W. Ballarotto, K. Siegrist, R.J. Phaneuf, E.D. Williams, W.-C. Yang, and R.J. Nemanich, Appl. Phys. Lett. 78, 3547 (2001).

${ }^{23}$ V.N. Litvinenko (unpublished).

${ }^{24}$ J. Ku and R.J. Nemanich, J. Appl. Phys. 80, 4715 (1996)

${ }^{25}$ R. Schmiedl, V. Demuth, P. Lahnor, H. Godehardt, Y. Bodschwinna, C. Harder, L. Hammer, H.-P. Strunk, M. Schulz, and K. Heinz, Appl. Phys. A: Mater. Sci. Process. 62, 223 (1996).

${ }^{26}$ H. Itoh, S. Narui, A. Sayama, and T. Ichinokawa, Phys. Rev. B 45, 11136 (1992).

${ }^{27}$ R.J. Nemanich, M.J. Thompson, W.B. Jackson, C.C. Tsai, and B.L. Stafford, J. Vac. Sci. Technol. B 1, 519 (1983).

${ }^{28}$ B.J. Keene, Surf. Interface Anal. 10, 367 (1987).

${ }^{29}$ H. Jeon, C.A. Sukow, J.W. Honeycutt, G.A. Rozgonyi, and R.J. Nemanich, J. Appl. Phys. 71, 4269 (1992).

${ }^{30}$ T.R. Anthony and H.E. Cline, J. Appl. Phys. 43, 2473 (1972).

${ }^{31}$ J.L. Bocquet, G. Brebec, and Y. Limoge, in Physical Metallurgy, edited by R.W. Chan (Elsevier, New York, 1983). 